\title{
Identifying recent Mycobacterium tuberculosis transmission in the setting of high HIV and TB burden
}

\author{
Junior Mutsvangwa, ${ }^{1}$ Kerry A Millington, ${ }^{2}$ Kathryn Chaka, ${ }^{1}$ Tafadzwa Mavhudzi, ${ }^{1}$ \\ Yin-Bun Cheung, ${ }^{3}$ Peter R Mason, ${ }^{1}$ Anthony E Butterworth, ${ }^{1}$ Elizabeth L Corbett, ${ }^{3}$ \\ Ajit Lalvani $^{2}$
}

${ }^{1}$ Biomedical Research and Training Institute, Harare, Zimbabwe

${ }^{2}$ Imperial College London, London, UK

${ }^{3}$ London School of Hygiene and Tropical Medicine, London, UK

\section{Correspondence to}

Professor Ajit Lalvani, Tuberculosis Research Unit, Department of Respiratory Medicine, National Heart and Lung Institute, Imperial College London, Norfolk Place, London W2 1PG, UK; a.lalvani@ imperial.ac.uk

JM and KAM contributed equally to this work.

Received 10 August 2009 Accepted 28 January 2010

\begin{abstract}
Background Accurate diagnosis of latent tuberculosis infection (LTBI) in recently exposed HIV-infected tuberculosis (TB) contacts is a public health priority because of the high risk of progression to active TB but is hampered by the high background prevalence of LTBI in high-burden populations and poor sensitivity of tuberculin skin testing (TST) in HIV co-infection.

Methods The prevalence of LTBI in 222 recent household contacts of TB cases and 176 household contacts of community controls without TB in Harare, Zimbabwe were compared using TST and interferon $\gamma$ enzyme-linked immunospot (ELISpot) responses to ESAT6 (early secretory antigenic target-6) and CFP-10 (culture filtrate protein-10). TST and ELISpot results were correlated with markers of recent TB exposure and the impact of HIV co-infection was assessed.
\end{abstract}

Results In this high-incidence population, the proportion of ELISpot-positive contacts was not significantly different from community controls. However, ELISpot, unlike TST, revealed a higher prevalence of LTBI in recent contacts of sputum smear-positive cases than in contacts of controls. ELISpot results correlated significantly with positive sputum smear and culture status of the index case (adjusted OR 2.40, $\mathrm{Cl} 1.12$ to 5.14), even in the subgroup of HIV-infected contacts (adjusted OR 5.36, Cl 1.11 to 25.93). and were independent of contacts' HIV status. TST results were also associated with positive smear and culture status of the index case (adjusted OR 4.41, $\mathrm{Cl} 1.82$ to 10.67) but were negatively associated with contacts' HIV status (adjusted OR 0.25, $\mathrm{Cl} 0.10$ to 0.60).

Conclusions Contact investigations in high-burden populations should focus on contacts of sputum smearpositive cases in whom recent infection can be detected by ELISpot, even in the presence of HIV co-infection.

\section{INTRODUCTION}

The devastating synergy between HIV and tuberculosis (TB) co-infection is a huge burden on public health especially in sub-Saharan Africa, to the extent that TB is the leading cause of death in HIVinfected patients. ${ }^{1}$ Treatment of latent tuberculosis infection (LTBI) in HIV-infected persons substantially decreases the risk of developing active $\mathrm{TB}^{2}{ }^{2}$ Because recently infected TB contacts and HIVinfected people are at especially high risk of developing active $\mathrm{TB}^{4}{ }^{5}$ accurately targeting those recently infected with Mycobacterium tuberculosis (MTB) is a high priority for improving TB control.

However, the high background prevalence of LTBI in the general population in high-burden settings makes it difficult to identify recently infected persons. Moreover, the sensitivity of the tuberculin skin test (TST) for diagnosis of LTBI is severely compromised in HIV-infected individuals. ${ }^{6}$ Specificity is also poor in BCG-vaccinated persons and in populations with high environmental mycobacterial exposure as in Africa. ${ }^{6}$ New blood tests (interferon $\gamma$ release assays (IGRAs)), which either measure the frequency of $\mathrm{T}$ cells secreting interferon $\gamma$ (IFN $\gamma$; enzyme-linked immunospot (ELISpot)) or the amount of IFN $\gamma$ released from whole blood (ELISA) in response to MTB-specific antigens absent from BCG and most environmental mycobacteria, have been developed. Given the absence of a gold standard test for LTBI, validation of these IGRAs for diagnosing LTBI has been based largely on the consistent correlation of test results with $\mathrm{TB}$ exposure in several studies in low and medium prevalence countries. ${ }^{8-15}$ However, in high $\mathrm{TB}$ prevalence countries, only three studies have investigated whether IGRAs are a valid marker of LTBI by correlating test results with TB exposure, ${ }^{16-18}$ and none has done so in HIVinfected TB contacts.

The diagnostic sensitivity of ELISpot in active $\mathrm{TB}^{19-22}$ and rates of positive IGRA results in persons undergoing HIV screening in a high TB prevalence country ${ }^{23}$ were unaffected by HIV coinfection; moreover, positive IGRA results in HIVinfected patients in Austria were recently shown to be prognostic of subsequent development of active TB. ${ }^{24}$ However, the utility of IGRAs for detecting LTBI in recent TB contacts in a population with a high prevalence of both HIV and TB has not hitherto been assessed. This is an important deficit in the current clinical evidence base since, along with child TB contacts, this is a large and growing population that stands to gain the greatest healthcare benefit from improved diagnosis of LTBI. We therefore addressed this clinical and epidemiological question in a population with extremely high burdens of both HIV and TB in sub-Saharan Africa. Our study was conducted in Harare, Zimbabwe, where the estimated prevalence of HIV infection among adults is $20 \%$ and the estimated prevalence rate of TB in 2006 was 597 per 100000 population. ${ }^{26}$ We tested for LTBI using ELISpot and TST, correlated test results with TB exposure in household contacts of TB cases and assessed the impact of HIV co-infection on test results in these contacts. On account of the high rates of MTB transmission in the general population we also assessed the prevalence of MTB infection in 
contacts of healthy community controls from households without TB cases.

\section{METHODS \\ Study participants}

Participants were recruited from February 2002 to November 2004 from within the framework of a larger longitudinal study, described elsewhere ${ }^{27}$ on the delivery of voluntary counselling and testing for HIV and a package of primary healthcare among factory workers in Harare, Zimbabwe. Small and medium sized enterprises in the two main industrial areas in Harare, covering a range of products, for example, food industry, textiles and telecommunications, were identified with the assistance of an HIV prevention project working with businesses (Zimbabwe AIDS Prevention Project), and were eligible if they had (1) 100-600 employees, (2) an occupational or first aid clinic and (3) individual-based absenteeism records. Index controls were randomly selected from the same payroll of the same factories as the index cases. Workers starting treatment for TB disease between January 2002 and November 2004 were invited to participate in this study along with their household contacts. Household contacts were all consenting individuals over the age of 10 years living with the TB cases. Contacts of controls were household contacts of workers with no evidence of TB disease randomly selected from the same factories as the TB cases; these contacts lived with the controls and were all consenting individuals over the age of 10 years.

\section{Tuberculin skin tests}

A two-step TST protocol was used to provide a suitable baseline for identifying subsequent TST conversions. ${ }^{28}{ }^{29}$ As recommended by the manufacturer, 2 units of RT-23 PPD (purified protein derivative) in Tween-80 (Statens Serum Institut, Copenhagen, Denmark) were injected intradermally into the forearm and results read at $48-72 \mathrm{~h}$. Placement and assessment followed recommended techniques. ${ }^{30}$ If the first reaction was $<10 \mathrm{~mm}$, then a second TST was placed after 7-14 days. Results were expressed as the greater of the two reactions. Reaction sizes $\geq 10 \mathrm{~mm}$ were considered positive.

\section{HIV testing}

Blood was drawn from individuals $>16$ years old for anonymous HIV testing at the time that the first TSTwas placed. Prevalence for children $12-16$ years old in Zimbabwe in 2003 was $0.86 \%$ (95\% CI 0.58 to 1.13; Sophie Pascoe and Frances Cowan, personal communication). Children aged 10-16 years were not tested and were assumed to be HIV negative. Serum samples were prepared and tested in parallel using Determine (Abbott, Wiesbaden, Germany) and Unigold (Trinity Biotech, Dunblane, UK). No discordant results were recorded. Voluntary counselling and testing was offered to all participants from whom HIV serology was requested for study purposes.

\section{ELISpot assays}

Blood was drawn for ELISpot testing before or after the TSTwas placed. ELISpot assays were carried out as described elsewhere. ${ }^{14}$ Duplicate wells contained no antigen (negative control), phytohaemagglutinin (positive control) (ICN Biomedical, Aurora, Ohio, USA) at $5 \mu \mathrm{g} / \mathrm{ml}$ or 13 pairs of duplicate wells each containing one of 13 peptide pools incorporating 5-7 overlapping 15-mer peptides spanning the length of early secretory antigenic target- 6 and culture filtrate protein-10, on which T-SPOT.TB is based. The final concentration of each peptide was $10 \mu \mathrm{g} / \mathrm{ml}$. ELISpot plates were sent to Oxford for automated spot counting (AID, Strassberg, Germany). Persons performing and reading the assays were blind to all personal identifiers and TST results.

\section{Data analysis}

Personal data, TST and HIV results were captured with EpiInfo 2002 (Centers for Disease Control, Atlanta, Georgia, USA) into ACCESS databases. Data were double-entered and checked. ELISpot counts were captured with EXCEL. We scored responses as positive if test wells contained a mean of at least five spotforming cells more than the mean of the negative control wells and were at least twice the mean of the negative control wells. This predefined cut-off point is the standard threshold used with our assay format in 10 studies including a total of 2700 participants. $^{8} 1214151920$ 31-34 Data analysis was carried out with STATA 9.0 (StataCorp, College Station, Texas, USA). Associations between categorical variables were tested for significance by $\chi^{2}$ or the Fisher exact method as appropriate, and $\kappa$ values were calculated for agreement between tests. Univariate and multivariate analysis of categorical values was carried out by logistic regression.

Written informed consent was obtained from all individuals, and from guardians of children $<18$ and $\geq 10$ years old. Children $<10$ years were not enrolled into the study. HIV tests were run and stored under dedicated laboratory numbers with no other personal identifiers.

\section{RESULTS}

\section{Characteristics of the study population}

We enrolled 129 factory workers treated for TB disease during the study period. For diagnosis, 104 underwent sputum smear microscopy for MTB by auramine fluorescence and Ziehl-Neelsen staining as well as sputum culture on LJ medium; 49 index cases had a positive MTB smear and culture, 18 cases had a negative smear but positive culture and 37 index cases were negative on both smear and culture testing. Twentyfive cases did not have a culture taken before TB treatment was started, but met clinical and radiological case definitions of $\mathrm{TB}$, including documented response to TB treatment after 1 month. Characteristics of the 129 index cases and their 222 household contacts and 149 index controls and their 176 contacts are shown in table 1. Factory workers diagnosed with TB disease were predominantly young or middle-aged adult males with a high $(77 \%)$ prevalence of HIV co-infection. Household contacts of index cases, representing mainly their spouses and children, were predominantly female, with a broader distribution of age. Children $<10$ years old did not undergo venepuncture for ELISpot assays and are not included in the study population. Children between 10 and 16 years old represented $27 \%$ and $24 \%$ of contacts of TB cases and controls, respectively, and were assumed to be HIV negative. HIV prevalence was significantly higher among household contacts of TB cases than among contacts of controls ( $25 \%$ vs $10 \%, \mathrm{p}<0.001)$. For both groups, household contacts were significantly more likely to be HIV positive if the index case was also positive (table 1). Eightysix per cent of our cohort had BCG scars.

\section{Prevalence of positive ELISpot and TST results in contacts and controls}

The prevalence of positive TST results was generally higher than the prevalence of positive ELISpot results in both contacts of index cases and contacts of community controls. Within each 
Table 1 Study group characteristics

\begin{tabular}{|c|c|c|c|c|c|c|c|}
\hline & \multicolumn{3}{|l|}{ Index cases } & \multicolumn{3}{|c|}{ Index controls } & \multirow[b]{2}{*}{ p Value $\dagger$} \\
\hline & HIV positive & HIV negative & p Value* & HIV positive & HIV negative & p Value* & \\
\hline $\mathrm{n}$ & $99(77 \%)$ & 30 & & $29(19 \%)$ & 120 & & $<0.001$ \\
\hline Number male (\%) & $95(96 \%)$ & $29(97 \%)$ & 1.00 & $25(90 \%)$ & $110(92 \%)$ & 0.48 & $<0.001$ \\
\hline \multirow[t]{3}{*}{ Age in years, median (range) } & $40(24-57)$ & $37(22-58)$ & 0.16 & $46(23-61)$ & $37(19,65)$ & 0.03 & 0.80 \\
\hline & \multicolumn{3}{|c|}{ Contacts of index cases } & \multicolumn{3}{|c|}{ Contacts of index controls } & \\
\hline & HIV positive & HIV negative $\ddagger$ & p Value* & HIV positive & HIV negative $\ddagger$ & p Value* & p Value $\S$ \\
\hline $\mathrm{n}$ & $55(25 \%)$ & 167 & & $18(10 \%)$ & 158 & & $<0.001$ \\
\hline Number male (\%) & $4(7 \%)$ & $65(39 \%)$ & $<0.001$ & $4(22 \%)$ & $64(41 \%)$ & 0.2 & $<0.001$ \\
\hline Age in years, median (range) & $30(17-47)$ & $19(10-82)$ & $<0.001$ & $30(22-48)$ & $21(10-77)$ & $<0.001$ & 0.95 \\
\hline \multicolumn{8}{|l|}{ HIV status of index case } \\
\hline Positive & 49 & 123 & & 8 & 16 & & \\
\hline Negative & 6 & 44 & 0.02 & 10 & 142 & $<0.001$ & 0.81 \\
\hline \multicolumn{8}{|l|}{ Smear status of index cases $\Phi$} \\
\hline Smear negative, culture negative & 4 & 13 & & - & - & & \\
\hline Smear negative, culture positive & 7 & 27 & & - & & & \\
\hline Smear positive, culture positive & 22 & 62 & & - & - & & \\
\hline
\end{tabular}

${ }^{*} p$ Values computed with Fisher exact test or test for trend.

$t p$ Value for difference between index cases and index controls.

$\neq$ Children $\leq 16$ years old were assumed to be HIV negative. They were not tested for their HIV status.

$\S p$ Value for difference between contacts of index cases and contacts of index controls.

I47 contacts were exposed to index cases that did not have a smear or culture taken.

contact group the prevalence of positive TST results was significantly lower in contacts infected with HIV than in HIVnegative contacts, whilst there was no significant difference in the prevalence of positive ELISpot results between HIV-positive and HIV-negative contacts (table 2). There was no significant difference in the prevalence of positive TST or ELISpot results between contacts of index cases compared with contacts of community controls, even when stratified by HIV status (table 2). However, a significantly higher proportion of contacts exposed to smear- and culture-positive index cases were ELISpot positive than contacts of controls (42/84 vs $53 / 176, p=0.002)$ whilst the proportion of TST-positive contacts exposed to smear- and culture-positive TB cases remained non-significantly different from contacts of controls (71/84 vs 132/176, $p=0.08$ ), reflecting the very high background rates of positive TST results in contacts of controls. TST did not boost responses in the

Table 2 TST and ELISpot results in contacts of TB cases and contacts of controls, and the impact of HIV infection

\begin{tabular}{|c|c|c|c|c|c|}
\hline & \multicolumn{2}{|c|}{ Contacts of patients with TB } & \multicolumn{2}{|c|}{ Contacts of controls } & \multirow{2}{*}{$\begin{array}{l}\text { p Value } \\
\text { contacts } \\
\text { vs controls* }\end{array}$} \\
\hline & $\begin{array}{l}\text { Positive } \\
\text { n (\%) }\end{array}$ & $\begin{array}{l}\text { p Value impact of } \\
\text { HIV co-infection }\end{array}$ & $\begin{array}{l}\text { Positive } \\
\text { n (\%) }\end{array}$ & $\begin{array}{l}\text { p Value impact of } \\
\text { HIV co-infection }\end{array}$ & \\
\hline \multicolumn{6}{|l|}{ TST results } \\
\hline All & $161(73)$ & $<0.001$ & $132(75)$ & $<0.001$ & 0.58 \\
\hline HIV positive & $27(49)$ & - & $6(33)$ & - & 0.29 \\
\hline HIV negative & $134(80)$ & - & $126(80)$ & - & 0.91 \\
\hline $\begin{array}{l}\text { Exposed to smear-negative and } \\
\text { culture-negative index cases }\end{array}$ & $34(60 \%)$ & - & - & - & - \\
\hline $\begin{array}{l}\text { Exposed to smear-negative and } \\
\text { culture-positive index cases }\end{array}$ & $23(68 \%)$ & - & - & - & - \\
\hline $\begin{array}{l}\text { Exposed to smear-positive } \\
\text { index cases }\end{array}$ & $71(85 \%)$ & - & - & - & - \\
\hline \multicolumn{6}{|l|}{ ELISpot results } \\
\hline All & $79(36)$ & 0.85 & $53(30)$ & 0.28 & 0.25 \\
\hline HIV positive & $19(35)$ & - & $3(17)$ & - & 0.24 \\
\hline HIV negative & $60(36)$ & - & $50(32)$ & - & 0.42 \\
\hline $\begin{array}{l}\text { Exposed to smear-negative and } \\
\text { culture-negative index cases }\end{array}$ & $16(28 \%)$ & - & - & - & \\
\hline $\begin{array}{l}\text { Exposed to smear-negative and } \\
\text { culture-positive index cases }\end{array}$ & $8(24 \%)$ & - & - & - & - \\
\hline $\begin{array}{l}\text { Exposed to smear-positive and } \\
\text { culture-positive index cases }\end{array}$ & $42(50 \%)$ & - & - & - & - \\
\hline
\end{tabular}


ELISpot assay as the proportion of contacts and controls positive to ELISpot did not differ statistically in those who had the TST before the ELISpot test compared with those that had the ELISpot before the TST test $(p=0.84)$.

\section{Association of ELISpot and TST results with amount of exposure to TB}

Table 3 shows univariate and multivariate analyses of factors associated with positive ELISpot and TST results among household contacts of index cases, using contacts of sputum smear- and culture-negative index cases as the reference group. In both univariate and multivariate analyses positive ELISpot results were significantly associated with age (multivariate $\mathrm{OR}$ 1.05, CI 1.02 to 1.09) and being a contact of a smear-positive, culture-positive index case (multivariate OR 2.40, CI 1.12 to 5.14). Moreover, positive ELISpot results specifically in HIVpositive household contacts were more strongly associated with TB exposure (multivariate OR 5.36, CI 1.11 to 25.93; table 3). ELISpot results in contacts were independent of their sex and HIV infection status, as well as of the HIV status of the index case. TST results among contacts were associated with smear and culture positivity of the index case (multivariate OR 4.41, CI 1.82 to 10.67; table 3) but were inversely associated with HIV status of the contact (multivariate OR 0.25 , CI 0.10 to 0.60 ). TST results were independent of HIV status of the index case and sex (multivariate OR 1.10, CI 0.44 to 2.75; OR 1.91, CI 0.72 to 5.05 , respectively).

\section{Impact of HIV infection on TST and ELISpot results}

Overall agreement between TST and ELISpot results was modest or poor ( $\kappa$ ranging from 0.15 to 0.19 , table 4 ). This poor agreement was mainly driven by the large number of discordant results in contacts of patients with TB and contacts of controls who were TST positive and ELISpot negative, probably reflecting the confounding effects of BCG vaccination and environmental mycobacterial exposure on TST. Agreement between TST and ELISpot results was substantially greater among HIV-positive individuals in each study group ( $\kappa$ ranging from 0.29 to 0.41 , table 4 ) than among HIV-negative individuals
( $\kappa$ ranging from 0.08 to 0.17 , table 4 ). These results suggest that the sensitivity of ELISpot is unaffected by HIV co-infection whereas the sensitivity of TST is significantly reduced.

In some countries $\mathrm{a} \geq 5 \mathrm{~mm}$ TST reaction is considered a positive result. ${ }^{30}$ Reducing the cut-off threshold for a positive result from 10 to $5 \mathrm{~mm}$ did not identify significantly more HIVpositive contacts or controls as being latently infected with $\mathrm{TB}$ ( $p=0.70$ and $p=0.49$, respectively). Reducing the cut-off value in active TB cases co-infected with HIV has also been found to be of limited benefit. ${ }^{35}$

Amongst the HIV-negative contacts and contacts of controls, there was a distinct non-zero distribution of TST induration peaking at $\sim 15-19$ and $10-14 \mathrm{~mm}$, respectively. In this HIVnegative population there was only a small proportion of nonreactors $(12 \%$ and $15 \%$, respectively). In contrast, there were a greater proportion of non-reactors amongst the HIV-positive contacts and contacts of controls (43\% and $56 \%$, respectively), but the overall distributions of TST indurations above zero were similar.

\section{DISCUSSION}

HIV-infected persons recently infected with MTB are at a very high risk of progressing to active $\mathrm{TB}^{4}$ and, given the large and growing global burden of HIV and $\mathrm{TB}$, prompt and accurate diagnosis of recently acquired LTBI in HIV-infected persons is a public health priority. Despite this, no studies have hitherto assessed the potential utility of IGRAs to detect recently infected close TB contacts in a setting of high HIV and TB prevalence. In such a setting, we found that the prevalence of positive ELISpot and TST results did not differ between household TB contacts and contacts of community controls as a whole, but there was a higher prevalence of positive ELISpot results in contacts specifically exposed to smear-positive index cases than in contacts of community controls. In recent household TB contacts positive ELISpot results were associated with TB exposure and, unlike TST, ELISpot results were not significantly adversely affected by HIV co-infection. Moreover, the relationship of positive ELISpot results to $\mathrm{TB}$ exposure

Table 3 Univariate and multivariate ORs of relationships between risk factors and $M$ tuberculosis infection as determined by ELISpot and TST among household contacts of index cases

\begin{tabular}{|c|c|c|c|c|c|}
\hline \multirow[b]{2}{*}{ Characteristic } & \multirow[b]{2}{*}{ Category } & \multicolumn{2}{|l|}{ ELISpot } & \multirow{2}{*}{$\begin{array}{l}\text { TST } \\
\text { Univariate } \\
\text { OR }(95 \% \text { CI) }\end{array}$} & \multirow[b]{2}{*}{$\begin{array}{l}\text { Multivariate } \\
\text { OR }(95 \% \mathrm{CI})\end{array}$} \\
\hline & & $\begin{array}{l}\text { Univariate } \\
\text { OR }(95 \% \mathrm{CI})\end{array}$ & $\begin{array}{l}\text { Multivariate } \\
\text { OR (95\% CI) }\end{array}$ & & \\
\hline \multicolumn{6}{|l|}{ All contacts* $n=175$} \\
\hline Age & & 1.05 (1.02 to 1.07$)$ & 1.05 (1.02 to 1.09$)$ & $0.98(0.95$ to 1.00$)$ & 0.99 (0.96 to 1.03$)$ \\
\hline \multirow[t]{2}{*}{ Sex } & Female & 1 & 1 & 1 & 1 \\
\hline & Male & $0.83(0.42$ to 1.63$)$ & $0.97(0.44$ to 2.14$)$ & $3.10(1.28$ to 7.48$)$ & $1.91(0.72$ to 5.05$)$ \\
\hline \multirow{3}{*}{$\begin{array}{l}\text { Smear and culture status } \\
\text { of index case }\end{array}$} & Smear negative, culture negative & 1 & 1 & 1 & 1 \\
\hline & Smear negative, culture positive & $0.79(0.30$ to 2.10$)$ & $0.73(0.25$ to 2.07$)$ & 1.41 (0.58 to 3.45$)$ & 1.36 (0.50 to 3.68$)$ \\
\hline & Smear positive, culture positive & $2.56(1.25$ to 5.26$)$ & $2.40(1.12$ to 5.14$)$ & 3.69 (1.67 to 8.17$)$ & 4.41 (1.82 to 10.67$)$ \\
\hline \multirow[t]{2}{*}{ HIV status of contacts } & Negative & 1 & 1 & 1 & 1 \\
\hline & Positive & 1.05 (0.52 to 2.13$)$ & $0.70(0.30$ to 1.62$)$ & $0.24(0.12$ to 0.49$)$ & $0.25(0.10$ to 0.60$)$ \\
\hline \multirow[t]{2}{*}{ HIV status of index cases } & Negative & 1 & 1 & 1 & 1 \\
\hline & Positive & $0.60(0.30$ to 1.17$)$ & 0.61 (0.28 to 1.31$)$ & $0.66(0.30$ to 1.47$)$ & $1.10(0.44$ to 2.75$)$ \\
\hline \multicolumn{6}{|l|}{ HIV-positive contacts $\dagger \mathrm{n}=44$} \\
\hline \multirow{3}{*}{$\begin{array}{l}\text { Smear and culture status } \\
\text { of index case }\end{array}$} & Smear negative, culture negative & 1 & 1 & 1 & 1 \\
\hline & Smear negative, culture positive & $1.6(0.20$ to 12.69$)$ & $1.87(0.22$ to 16.16$)$ & $1.5(0.24$ to 9.46$)$ & 1.09 (0.13 to 9.42$)$ \\
\hline & Smear positive, culture positive & $4.8(1.05$ to 21.91$)$ & 5.36 (1.11 to 25.93$)$ & $3.5(0.88$ to 13.93$)$ & $3.43(0.76$ to 15.52$)$ \\
\hline
\end{tabular}

* 47 contacts exposed to the 25 index cases that did not have both a smear and culture taken have been excluded from this analysis.

$\dagger 11 / 47$ contacts exposed to the 25 index cases that did not have both a smear and culture taken and excluded from this analysis were HIV positive.

ELISpot, enzyme-linked immunospot; TST, tuberculin skin testing. 
Table 4 Agreement between TST and ELISpot results and impact of HIV infection

\begin{tabular}{|c|c|c|c|c|c|c|c|c|c|}
\hline & & \multicolumn{4}{|c|}{ Contacts of patients with TB } & \multicolumn{4}{|c|}{ Contacts of controls } \\
\hline & & ELISpot+ & ELISpot- & $\%$ ELISpot+ & $\kappa$ & ELISpot+ & ELISpot- & \% ELISpot+ & $\kappa$ \\
\hline \multirow[t]{2}{*}{ Overall } & TST+ & 67 & 94 & 42 & 0.15 & 50 & 82 & 38 & 0.19 \\
\hline & TST- & 12 & 49 & 20 & $p=0.001$ & 3 & 41 & 7 & $\mathrm{p}<0.001$ \\
\hline \multirow[t]{2}{*}{ HIV+ } & TST+ & 15 & 12 & 56 & 0.41 & 2 & 4 & 33 & 0.29 \\
\hline & TST- & 4 & 24 & 14 & $\mathrm{p}<0.001$ & 1 & 11 & 8 & $p=0.09$ \\
\hline HIV- & TST- & 8 & 25 & 24 & $p=0.06$ & 2 & 30 & 6 & $\mathrm{p}<0.001$ \\
\hline
\end{tabular}

ELISpot, enzyme-linked immunospot; TB, tuberculosis; TST, tuberculin skin testing.

within households remained strongly significant in the subgroup of HIV-positive contacts. These findings suggest a means for accurate diagnostic assessment of persons at highest risk of progression to $\mathrm{TB}$ in communities with a high burden of $\mathrm{TB}$ and HIV.

Although association of IGRA results with TB exposure in low prevalence countries is now well established, ${ }^{8-15}$ only three other studies have investigated whether IGRA results are associated with TB exposure in a high TB prevalence setting. ${ }^{16-18}$ The prevalence of HIV in these studies was low. Ours is the first study to demonstrate that IGRA results correlate with recent TB exposure in the most vulnerable group, HIV-infected close contacts.

There was a significant increase in positive ELISpot results with age, consistent with the cumulative risk of acquiring LTBI with increasing age and ongoing $\mathrm{TB}$ exposure and transmission in high prevalence areas with a high annual risk of infection. This is in contrast to a contact tracing study in a low prevalence country which found that TST results but not IGRA results were associated with age. ${ }^{36}$ Positive TST results in the older individuals in that study were probably due to remotely acquired LTBI whereas the increasing prevalence of LTBI with age in our population probably reflects a mixture of both remote and recently acquired LTBI.

Our observation that the proportion of household contacts that were ELISpot positive was not significantly higher than the proportion of contacts of controls is also consistent with high levels of $\mathrm{TB}$ exposure and transmission in the community outside the households of TB cases. ${ }^{37}$ However, among the household contacts exposed to smear- and culture-positive index cases, a significantly higher proportion had positive ELISpot results than did contacts of controls, suggesting that even in an ultrahigh prevalence setting, ELISpot detects contacts with significant recent TB exposure. Thus, in a high-burden setting testing for recently acquired LTBI should be targeted at close contacts of sputum- and smear-positive index cases.

ELISpot results were not significantly different in HIVinfected compared with HIV-uninfected household contacts. In contrast, the proportion of TST-positive contacts was significantly lower in HIV-infected contacts compared with HIVuninfected contacts. Unlike TST, ELISpot results in the contacts of community controls were also unaffected by HIV status, similar to the observation that rates of positive ELISpot results and, to a lesser extent, ELISA results were robust to HIV infection in South African adults undergoing screening for HIV infection. ${ }^{23}$ CD4 counts of the HIV-infected participants of this study were not determined. Although to date ELISpot results have been shown to be independent of CD4 T cell counts in patients with HIV-1,22 3839 it is possible that very low CD4 counts might adversely affect ELISpot results.
The prevalence of HIV in household contacts was significantly higher than among contacts of controls. This may be explained by the fact that contacts of both patients with $\mathrm{TB}$ and controls were significantly more likely to be HIV positive if the index case or control were also positive, and the prevalence of HIV in the index cases was significantly higher than the prevalence in the controls. These very high rates of HIV co-infection are comparable with those reported in another study in Harare. ${ }^{40}$ Moreover, most of the contacts of index cases were spouses. Eighty-six per cent of our cohort had BCG scars, similar to the proportion of BCG scars observed in 1997 when BCG coverage was $96.3 \%$. ${ }^{41}$ We did not enrol children $\leq 10$ years old because of the cultural challenges with venepuncture of small children in this population. Although child TB contacts in low ${ }^{12-14}$ and high ${ }^{16}$ prevalence countries have been studied, large-scale assessment of IGRAs in HIV-infected child TB contacts remains a priority.

In contacts of patients with $\mathrm{TB}$ and in contacts of controls, positive TST results were frequently associated with negative ELISpot responses, consistent with poor specificity of the TST in BCG-vaccinated populations with environmental mycobacterial exposure. These factors may be largely responsible for the poor level of agreement between ELISpot and TST results, particularly in HIV-negative persons, among whom the prevalence of positive TST results was uniformly high.

Targeting preventive therapy to TST-positive HIV-infected adults reduces the risk of active TB in these individuals in the short to medium term. ${ }^{3}$ Research into the long-term effects along with the impact on mortality conducted in large trials is required. Our findings suggest that ELISpot is a more accurate test than TST in HIV-infected persons recently infected with TB in a high-burden setting for both these infections. The increased accuracy of ELISpot testing compared with TST could improve targeting of preventive treatment to HIV-infected recent contacts of TB with LTBI which could further reduce the risk of active $\mathrm{TB}$. The relevance of our findings for influencing $\mathrm{TB}$ control strategies was recently underscored by the demonstration that positive IGRA results in recent TB contacts are prognostic of development of active TB disease. ${ }^{42} 43$ Prospective trials of $\mathrm{TB}$ clinical outcomes in recent contacts given preventive treatment on the basis of IGRA results are now warranted to determine whether this strategy can improve TB control, especially in sub-Saharan Africa.

Acknowledgements We thank the factory workers and their contacts for their participation.

Funding This study was funded by the Wellcome Trust. ELC is supported by a Wellcome Trust Senior Fellowship in Clinical Tropical Medicine and AL is supported by a Wellcome Trust Senior Research Fellowship in Clinical Science and a National Institute of Health Research Senior Investigator Award. The Wellcome Trust had no role in the design and conduct of the study; collection, management, analysis, and interpretation of the data; and preparation, review, or approval of the manuscript. 
Competing interests $\mathrm{AL}$ and KAM are inventors of patents relating to $\mathrm{T}$ cell-based diagnosis. The Lalvani ELISpot was commercialised by an Oxford University spin-out company (T-SPOT.TB, Oxford Immunotec, Abingdon, UK) in which Oxford University and $\mathrm{AL}$ have minority shares of equity and to which $\mathrm{AL}$ acted as non-executive director from 2003 to 2007. All other authors declare that they have no conflict of interest.

Ethics approval The study was approved by the ethics committees of the London School of Hygiene and Tropical Medicine, the Biomedical Research and Training Institute and the Medical Research Council of Zimbabwe.

Contributors $A B, E C$ and $A L$ designed the study. EC enrolled and clinically assessed the contacts and created the clinical and demographic database. JM and KC performed the ELISpot assays. KM read the ELISpot assay results. JM, YBC and AB unblinded and combined the clinical and ELISpot databases. The analysis was designed by $A B, E C$ and $A L$, and the statistical analysis was done by $Y B C$ and $K M$. $J M, A B, K M$ and $A L$ wrote the paper. All researchers reviewed the final report.

Provenance and peer review Not commissioned; externally peer reviewed.

\section{REFERENCES}

1. Corbett EL, Marston B, Churchyard GJ, et al. Tuberculosis in sub-Saharan Africa: opportunities, challenges, and change in the era of antiretroviral treatment. Lance 2006; $\mathbf{3 6 7}: 926-37$

2. Whalen CC, Johnson JL, Okwera A, et al. A trial of three regimens to prevent tuberculosis in Ugandan adults infected with the human immunodeficiency virus. Uganda-Case Western Reserve University Research Collaboration. N Engl J Med 1997;337:801-8.

3. Woldehanna S, Volmink J. Treatment of latent tuberculosis infection in HIV infected persons. Cochrane Database Syst Rev 2004(1):CD000171.

4. Selwyn PA, Hartel D, Lewis VA, et al. A prospective study of the risk of tuberculosis among intravenous drug users with human immunodeficiency virus infection. $N$ Engl $J$ Med 1989;320:545-50.

5. Sonnenberg $\mathbf{P}$, Glynn JR, Fielding $\mathrm{K}$, et al. How soon after infection with HIV does the risk of tuberculosis start to increase? A retrospective cohort study in South African gold miners. J Infect Dis 2005;191:150-8.

6. Reid MJ, Shah NS. Approaches to tuberculosis screening and diagnosis in people with HIV in resource-limited settings. Lancet Infect Dis 2009;9:173-84.

7. Lavlani A, Pareek M. 100-year update on diagnosis of tuberculosis infection. Br Med Bull 2009[Epub ahead of print].

8. Soysal A, Millington KA, Bakir M, et al. Effect of BCG vaccination on risk of Mycobacterium tuberculosis infection in children with household tuberculosis contact: a prospective community-based study. Lancet 2005;366:1443-51.

9. Shams H, Weis SE, Klucar P, et al. Enzyme-linked immunospot and tuberculin skin testing to detect latent tuberculosis infection. Am J Respir Crit Care Med 2005;172:1161-8.

10. Zellweger JP, Zellweger $A$, Ansermet $\mathrm{S}$, et al. Contact tracing using a new T-cellbased test: better correlation with tuberculosis exposure than the tuberculin skin test. Int J Tuberc Lung Dis 2005;9:1242-7.

11. Kang YA, Lee HW, Yoon HI, et al. Discrepancy between the tuberculin skin test and the whole-blood interferon gamma assay for the diagnosis of latent tuberculosis infection in an intermediate tuberculosis-burden country. JAMA 2005:293:2756-61.

12. Richeldi L, Ewer K, Losi M, et al. T cell-based tracking of multidrug resistant tuberculosis infection after brief exposure. Am J Respir Crit Care Med 2004:170:288-95

13. Brock I, Weldingh K, Lillebaek T, et al. Comparison of tuberculin skin test and new specific blood test in tuberculosis contacts. Am J Respir Crit Care Med 2004:170:65-9.

14. Ewer K, Deeks J, Alvarez L, et al. Comparison of T-cell-based assay with tuberculin skin test for diagnosis of Mycobacterium tuberculosis infection in a school tuberculosis outbreak. Lancet 2003;361:1168-73

15. Lalvani A, Pathan AA, Durkan $\mathrm{H}$, et al. Enhanced contact tracing and spatial tracking of Mycobacterium tuberculosis infection by enumeration of antigen-specific $T$ cells. Lancet 2001;357:2017-21.

16. Hill PC, Brookes RH, Fox A, et al. Large-scale evaluation of enzyme-linked immunospot assay and skin test for diagnosis of Mycobacterium tuberculosis infection against a gradient of exposure in The Gambia. Clin Infect Dis 2004;38:966-73.

17. Nakaoka H, Lawson L, Squire SB, et al. Risk for tuberculosis among children. Emerg Infect Dis 2006;12:1383-8.
18. Okada K, Mao TE, Mori T, et al. Performance of an interferon-gamma release assay for diagnosing latent tuberculosis infection in children. Epidemiol Infect 2008:136:1179-87.

19. Chapman $\mathbf{A L}$, Munkanta M, Wilkinson KA, et al. Rapid detection of active and laten tuberculosis infection in HIV-positive individuals by enumeration of Mycobacterium tuberculosis-specific T cells. AIDS 2002;16:2285-93.

20. Liebeschuetz S, Bamber S, Ewer K, et al. Diagnosis of tuberculosis in South African children with a T-cell-based assay: a prospective cohort study. Lancet 2004:364:2196-203.

21. Bourgarit A, Carcelain G, Martinez V, et al. Explosion of tuberculin-specific Th1responses induces immune restoration syndrome in tuberculosis and HIV co-infected patients. AIDS 2006;20:F1-7.

22. Clark SA, Martin SL, Pozniak A, et al. Tuberculosis antigen-specific immune responses can be detected using enzyme-linked immunospot technology in human immunodeficiency virus (HIV)-1 patients with advanced disease. Clin Exp Immunol 2007;150:238-44.

23. Rangaka MX, Wilkinson KA, Seldon R, et al. Effect of HIV-1 infection on T-cell-based and skin test detection of tuberculosis infection. Am J Respir Crit Care Med 2007; 175:514-20.

24. Aichelburg MC, Rieger A, Breitenecker $F$, et al. Detection and prediction of active tuberculosis disease by a whole-blood interferon-gamma release assay in HIV-1infected individuals. Clin Infect Dis 2009;48:954-62.

25. Gregson S, Garnett GP, Nyamukapa CA, et al. HIV decline associated with behavio change in eastern Zimbabwe. Science 2006;311:664-6.

26. World Health Organization. Global tuberculosis control: surveillance, planning financing. Geneva, Switzerland: WHO, 2008, Report No: WHO/HTM/TB/2008.393.

27. Corbett EL, Dauya E, Matambo R, et al. Uptake of workplace HIV counselling and testing: a cluster-randomised trial in Zimbabwe. PLoS Med 2006;3:e238.

28. Menzies D. Interpretation of repeated tuberculin tests. Boosting, conversion, and reversion. Am J Respir Crit Care Med 1999;159:15-21.

29. Bass JA Jr., Serio RA. The use of repeat skin tests to eliminate the booste phenomenon in serial tuberculin testing. Am Rev Respir Dis 1981;123:394-6.

30. American Thoracic Society. Targeted tuberculin testing and treatment of latent tuberculosis infection. Am J Respir Crit Care Med 2000:161:S221-47.

31. Lalvani A, Pathan AA, McShane $\mathrm{H}$, et al. Rapid detection of Mycobacterium tuberculosis infection by enumeration of antigen-specific T cells. Am J Respir Crit Care Med 2001;163:824-8.

32. Pathan AA, Wilkinson KA, Klenerman $P$, et al. Direct ex vivo analysis of antigenspecific IFN-gamma-secreting CD4 T cells in Mycobacterium tuberculosis-infected individuals: associations with clinical disease state and effect of treatment. $J$ Immunol 2001;167:5217-25.

33. Lalvani A, Nagvenkar P, Udwadia Z, et al. Enumeration of T cells specific for RD1encoded antigens suggests a high prevalence of latent Mycobacterium tuberculosis infection in healthy urban Indians. J Infect Dis 2001;183:469-77.

34. Dosanjh DP, Hinks TS, Innes JA, et al. Improved diagnostic evaluation of suspected tuberculosis. Ann Intern Med 2008;148:325-36.

35. Cobelens FG, Egwaga SM, van Ginkel T, et al. Tuberculin skin testing in patients with HIV infection: limited benefit of reduced cutoff values. Clin Infect Dis 2006:43:634-9.

36. Arend SM, Thiijsen SF, Leyten EM, et al. Comparison of two interferon-gamma assays and tuberculin skin test for tracing tuberculosis contacts. Am J Respir Crit Care Med 2007;175:618-27.

37. Madico G, Gilman RH, Checkley W, et al. Community infection ratio as an indicator for tuberculosis control. Lancet 1995;345:416-19.

38. Karam F, Mbow F, Fletcher $\mathrm{H}$, et al. Sensitivity of IFN-gamma release assay to detect latent tuberculosis infection is retained in HIV-infected patients but dependent on HIV/AIDS progression. PLOS ONE 2008;3:e1441.

39. Lawn SD, Bangani N, Vogt M, et al. Utility of interferon-gamma ELISPOT assay responses in highly tuberculosis-exposed patients with advanced HIV infection in South Africa. BMC Infect Dis 2007;7:99

40. Corbett EL, Bandason T, Cheung YB, et al. Epidemiology of tuberculosis in a high HIV prevalence population provided with enhanced diagnosis of symptomatic disease. PLoS Med 2007;4:e22.

41. Sikhosana P. Family and child health department, Executive Summary: Government of Zimbabwe, 1998:1005.

42. Bakir M, Millington KA, Soysal A, et al. Prognostic value of a T cell based interferon-gamma biomarker in child tuberculosis contacts. Ann Intern Med 2008; 149:777-86.

43. Diel R, Loddenkemper R, Meywald-Walter K, et al. Predictive value of a whole-blood IFN-\{gamma $\}$ assay for the development of Active TB disease. Am J Respir Crit Care Med 2008;177:1164-70. 